\title{
Model Predictive Control Strategies Performance Evaluation over a Pipeline Transportation System
}

\author{
Jorge Cardenas-Cabrera, ${ }_{1}^{1}$ Luis Diaz-Charris, ${ }^{1}$ Andrés Torres-Carvajal, ${ }^{1}$ \\ Narciso Castro-Charris, ${ }^{1}$ Elena Romero-Fandiño, ${ }^{2}$ José David Ruiz Ariza, ${ }^{1}$ \\ and Javier Jiménez-Cabas $\mathbb{D}^{1}$ \\ ${ }^{1}$ Departamento de Ciencias de la Computación y Electrónica, Universidad de la Costa, Calle 58 \#55-66., Barranquilla, Colombia
${ }^{2}$ Departamento de Ingeniería Mecánica, Universidad del Norte, $\mathrm{km} .5$ Vía Puerto Colombia,
Área Metropolitana de Barranquilla, Colombia \\ Correspondence should be addressed to Javier Jiménez-Cabas; jajimenez8@hotmail.com
}

Received 23 January 2019; Accepted 9 April 2019; Published 20 May 2019

Academic Editor: Benoit Iung

Copyright (C) 2019 Jorge Cardenas-Cabrera et al. This is an open access article distributed under the Creative Commons Attribution License, which permits unrestricted use, distribution, and reproduction in any medium, provided the original work is properly cited.

In several industries using pipelines to transport different products from one point to another is a common and indispensable process, especially at oil/hydrocarbon industries. Thus, optimizing the way this process is carried out must be an issue that cannot be stopped. Therefore, the performance of the control strategy implemented is one way of reaching such optimal operating zones. This study proposes using Model Predictive Control strategies for solving some issues related to the proper operation of pipelines. It is proposed a model based on physics and thermodynamic laws, using MATLAB $^{\circledR}$ as the development environment. This model involves four pumping stations separated by three pipeline sections. Three MPC strategies are developed and implemented. Accordingly, the results indicate that a centralized controller with an antiwindup back-calculation method has the best results among the three configurations used.

\section{Introduction}

Transporting various fluids through pipelines is one of the most used transport mechanisms worldwide, mainly because it allows transporting large volumes in relatively short periods [1]. It is estimated that there are more than $3.500 .000(\mathrm{~km})$ of pipelines in about 120 countries of the world and around $134.866(\mathrm{~km})$ of pipelines are planned and under construction worldwide [2]. Given the fatalities and severe environmental and economic consequences of potential failures or downtime, pipeline transportation systems have high requirements regarding safety, reliability, and profitability. Thus, it is an ongoing concern for pipeline system operators to guarantee increasingly better performance.

Some works have been performed by researchers to enhance the performance of pipeline transportation systems $[3,4]$ by introducing a set of sufficient conditions to guarantee asymptotic stability for hydraulic pipeline systems described by the so-called water hammer equations. These conditions are then integrated into a receding horizon optimal control scheme. Although the proposed approach was validated in simulations, the dynamics of essential elements as sensors and actuators were not taken into account. Yuzhanin et al. [5] addressed the problem of maintaining pressure below the maximum rated load capacity in a linear section of an oil pipeline. The proposed algorithm can suggest the automatic control action that will allow dampening potentially dangerous pressure surges in the pipeline. The simulations results show an efficient performance of the algorithm. Osiadacz and Chaczykowski [6] described an algorithm for optimal control of a gas network. Since the compressors station, which forms a significant part of the operational plant of a gas transportation system, is driven by gas turbines which use natural gas as fuel, authors considered optimality concerning fuel consumption. Thus, minimizing this fuel usage is a primary objective in the optimal control problem considered. 
A PLC-based Fuzzy-PID controller is proposed in [7] to regulate the flow rate of the petroleum products at destination by controlling the multiple pressure signals range along the pipeline. Unlike the previously mentioned cases, whose results were tested through simulations, the performance of the Fuzzy-PID is assessed in a lab-scale experimental setup. Real-time experimental results showed that Fuzzy-PID controller provides better performance than a Cascade-PID controller.

Motivated by the fact that model predictive control (MPC) is the industry chosen technology for advanced control in many processes even over other advanced process control techniques such as constraint control, nonlinear control and fuzzy logic control [8]; in this work, its performance is studied on a pipeline transportation systems. MPC control strategies are based on using a model to predict process behavior shortly (moving horizon window), and then the future manipulated variable trajectory is computed over the same horizon by solving a constrained optimization problem. Only the first sample of the calculated trajectory is applied to the actuator, and the horizon window is moved one sample forward, and the optimization process is repeated using new measurement data. Most popular MPC strategies found in literature are DMC (Dynamic Matrix Control) [9], GPC (Generalized Predictive Control) [10], IMC (Internal Model Control) [11], RHC (Receding Horizon Control) [4], NMPC (Nonlinear Model Predictive Control) [12, 13], among others [14].

The essential contribution of this work is to explore and to implement in simulation three different MPC control configurations for a pipeline transportation process: (1) two decoupled control systems, an MPC based control system for pressure and an on-off control system for level, (2) two decoupled control systems for pressure and level based on MPC strategy, and (3) one MPC control system for pressure and level. Additionally, it has been proposed an alternative option for constraint handling which involves applying a back-calculation scheme. The addition of back-calculation as an antiwindup method allows reducing the control algorithms execution time significantly while maintaining the same performance as the achieved with the optimization based constraint handling method.

The rest of the paper is organized in the following pattern. Section 2 presents the proposed linear model that describes the fluid behavior in a pipeline and its validation by using data of a real pipeline used for transporting Liquefied Petroleum Gas. On the other hand, models for instrumentation and additional systems are presented too. Section 3 exposes the used Discrete Model Predictive Control strategy and the back-calculation scheme proposed as constrains handling method. Section 4, on the one hand, presents a comparison between the performances of the three control configurations proposed. Finally, Section 5 provides some conclusions.

\section{Fluid Dynamics Modeling}

2.1. Pipeline Model. By assuming negligible convective changes in velocity, as well as constant cross-sectional area, the momentum and continuity equations governing the dynamics of the fluid in a horizontal pipeline can be expressed as [15-18]:

$$
\begin{array}{r}
\frac{d Q(t, z)}{d t}+\frac{A_{P}}{\rho(t, z)} \frac{d P(t, z)}{d z}+\frac{f Q(t, z)|Q(t, z)|}{2 \phi A_{P}}=0 \\
\frac{d P(t, z)}{d t}+\frac{\rho(t, z) a^{2}}{A_{P}} \frac{d Q(t, z)}{d z}=0
\end{array}
$$

where $(z, t) \in\left(0, L_{p}\right) \times(0, \infty)$ gathers the space $(m)$ and time $(s)$ coordinates, respectively, $L_{p}$ is the length of the pipe, $P$ is the pressure $(\mathrm{Pa}), Q$ is the flow rate $\left(\mathrm{m}^{3} / \mathrm{s}\right), f$ is the Darcy-Weisbach friction factor, $A_{P}$ is the cross-sectional area of the pipe $\left(\mathrm{m}^{2}\right), \phi$ is the inside diameter of the pipe $(m), a$ is the wave speed in the fluid $(\mathrm{m} / \mathrm{s})$, and $\rho$ is the fluid density.

Due complexity to solve this set of equations, several linearized versions of this set have been proposed [19-22]. At the current study, the linearized model (3) is used to design control strategies.

$$
\begin{gathered}
{\left[\begin{array}{c}
P_{L_{P}} \\
Q_{0} \\
\rho_{L_{P}}
\end{array}\right](s)} \\
=\left[\begin{array}{ccc}
\frac{1}{\cosh \left(n L_{P}\right)} & -Z_{K} \tanh \left(n L_{P}\right) & -\frac{Q(0, t) T}{A_{P} s}\left(\frac{1}{\cosh \left(n L_{P}\right)}-e^{-\tau s}\right) \\
\frac{1}{Z_{K} \tanh \left(n L_{P}\right)} & \frac{1}{\cosh \left(n L_{P}\right)} & -\frac{Q(0, t) T}{Z_{K} A_{P} s} \tanh \left(n L_{P}\right) \\
0 & 0 \\
& \cdot\left[\begin{array}{c}
P_{0} \\
Q_{L_{P}} \\
\rho_{0}
\end{array}\right](s)
\end{array}\right]
\end{gathered}
$$


where $Z_{K}=\sqrt{(L s+R) / C s} R=\rho f|Q| / \phi A_{P} C=1 / \rho a^{2} T=$ $\lambda Q|Q| / 2 \phi A_{P}^{2} L=\rho / A_{P}, n=\sqrt{(L s+R) C s}$, and the subindexes 0 and $L_{P}$ indicate input and output.

In this work, the Liquefied Petroleum Gas (LPG) pipeline presented in [22] would be considered (see Figure 1).

The physical parameters of the pipeline are shown in Table 1.

LPG is a flammable mixture of hydrocarbon (primarily propane and butane), gaseous at standard temperature $\left(15^{\circ} \mathrm{C}\right)$ and pressure (0.1MPa), but easily liquefied by increasing pressure (see Figure 2). Due to its ease of handling, cleanness of combustion, high purity, and high heating values; LPG is used as fuel in a large variety of industrial, commercial and domestic uses [23].

2.2. Instrumentation and Additional Systems. In order to get closer to a realistic situation, the pipeline model was enriched with models of storage tanks, sensors/transmitters, and actuators. As it refers to fuel storage modeling, membrane tanks are commonly used. These tanks are designed to provide a constant inside pressure, which avoids phase changes in the system. Inlet and outlet taps in the tanks are positioned at the same height in order to have pressure changes only caused by the liquid column. The dynamic model for this kind of systems can be expressed as

$$
\frac{d h(t)}{d t}=\frac{1}{A_{T}}\left(Q_{\text {in }}(t)-Q_{\text {out }}(t)\right)
$$

where $h(t)$ is the level in the tank in $(m), A_{T}$ is the crosssectional area of the tank $\left(m^{2}\right), Q_{i n}$ and $Q_{o u t}$ are the flows of liquid into and from the tank, respectively $\left(\mathrm{m}^{3} / \mathrm{s}\right)$.

On the other hand, first-order models were considered for instruments (sensors/transmitters and actuators). There were added centrifugal pumps at the beginning of each pipeline section, control valves at each tank's inlet section, and level transmitters at each tank, as well as manual valves. Figure 3 shows the piping and instrumentation diagram (P\&ID) for a pipeline section. The total system is constituted by three of the sections shown in Figure 3.

Table 2 shows critical parameters of the instruments used to model its dynamical behavior. Taking into account the pipeline and instruments parameters, the obtained model corresponds to

$$
\underbrace{\left[\begin{array}{l}
P L_{1} \\
P L_{2} \\
P L_{3}
\end{array}\right]}_{C_{P L}}=\underbrace{\left[\begin{array}{ccc}
G_{11}(s) & 0 & 0 \\
G_{21}(s) & G_{22}(s) & 0 \\
G_{31}(s) & G_{32}(s) & G_{33}(s)
\end{array}\right]}_{G_{P L}(s)} \underbrace{\left[\begin{array}{c}
M_{p 1} \\
M_{p 2} \\
M_{p 3}
\end{array}\right]}_{M_{p}}
$$

where $P L_{i}$ is the pressure of pipeline section $i$ at the outlet and $M_{p i}$ is pressure drop in pump $i$. The form of the model representing the effect of valves position, $M_{v i}$ on the pressure at outlet section of each pipeline section, is represented in

$$
\underbrace{\left[\begin{array}{c}
P L_{1} \\
P L_{2} \\
P L_{3}
\end{array}\right]}_{C_{P L}}=\underbrace{\left[\begin{array}{ccc}
G_{14}(s) & 0 & 0 \\
G_{24}(s) & G_{25}(s) & 0 \\
G_{34}(s) & G_{35}(s) & G_{36}(s)
\end{array}\right]}_{G_{d}(s)} \underbrace{\left[\begin{array}{c}
M_{v 1} \\
M_{v 2} \\
M_{v 3}
\end{array}\right]}_{M_{v}}
$$

Moreover, the model that links valve position and tanks level takes the form shown in (7). Finally, it is important to mention manipulated $\left(M_{p}, M_{v}\right)$, and controlled variables $\left(C_{P L}, C_{L}\right)$ move in the range of 0 to $100 \%$.

$$
\underbrace{\left[\begin{array}{l}
H_{1} \\
H_{2} \\
H_{3}
\end{array}\right]}_{C_{L}}=\underbrace{\left[\begin{array}{ccc}
G_{44}(s) & G_{45}(s) & 0 \\
0 & G_{55}(s) & G_{56}(s) \\
0 & 0 & G_{66}(s)
\end{array}\right]}_{G_{L}(s)} \underbrace{\left[\begin{array}{c}
M_{v 1} \\
M_{v 2} \\
M_{v 3}
\end{array}\right]}_{M_{v}}
$$

By combining (5), (6), and (7) one obtains the perturbed system form

$$
\begin{aligned}
C_{P L}(s) & =G_{P L}(s) M_{p}(s)+G_{d}(s) M_{v}(s) \\
C_{L}(s) & =G_{L}(s) M_{v}(s)
\end{aligned}
$$

Discrete-time state space models that approximate the continuous-time models (8) were computed, with sampling time 1s.

$$
\begin{gathered}
G_{P L}(z)=\left[\begin{array}{c|c}
A_{12 \times 12}^{P L} & B_{12 \times 3}^{P L} \frac{1}{2} \\
\hline \frac{1}{2} C_{3 \times 12}^{P L} & 0_{3 \times 3}
\end{array}\right] \\
G_{d}(z)=\left[\begin{array}{c|c}
A_{12 \times 12}^{d} & B_{12 \times 3}^{d} \frac{1}{2} \\
\hline \frac{1}{2} C_{3 \times 12}^{d} & 0_{3 \times 3}
\end{array}\right] \\
G_{L}(z)=\left[\begin{array}{c|c}
A_{10 \times 10}^{L} & B_{10 \times 3}^{L} \\
\hline \frac{1}{2} C_{3 \times 10}^{L} & 0_{3 \times 3}
\end{array}\right]
\end{gathered}
$$

In (9) it shows the matrix dimensions for each model, where $G(z)=\left[\begin{array}{l|l}A & B \\ \hline C & D\end{array}\right]=C(z I-A)^{-1} B+D$.

\section{Implemented Control Strategy}

By increasing pressures, above the bubble curve for a given temperature or density (see Figure 2), LPG can be held in the liquid phase, which simplifies transporting, measuring, and storage. Once pressure is released, LPG vaporizes and becomes in an ideal energy source for fuel. In order to maintain LPG the liquid phase, tight pressure control is necessary to avoid possible phase changes during the transportation process. Additionally, it is well known that oscillations and rapid changes of pressure within the pipeline can cause undesirable phenomena as cavitation, slugging and hydraulic shock (water hammer). Then, these aspects must be considered in the control strategies performance analysis.

3.1. Discrete Model Predictive Control Strategy. The functionbased discrete time model predictive control (DMPC) formulation proposed by Wang [24] was applied to the LPG pipeline. The DMPC strategy considered is based on state space models and was implemented to control the downstream pressure of each pipeline section $\left(C_{P L}\right)$ and the liquid 


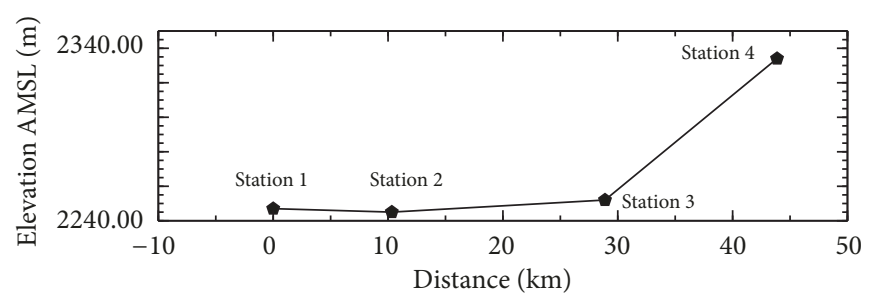

Figure 1: Liquefied Petroleum Gas pipeline.

TABle 1: Pipeline model parameters.

\begin{tabular}{lccccccc}
\hline Variable & Symbol & Value & Units & Variable & Symbol & Value & Units \\
\hline Density & $\rho$ & 530 & $\mathrm{Kg} / \mathrm{m}^{3}$ & Pipelines Diameter & $d$ & 0.5 & $\mathrm{~m}$ \\
\hline Friction Coefficient & $\lambda$ & 0.00965 & & Tanks Height & $H_{\max }$ & 15.7920 & $\mathrm{~m}$ \\
\hline Fluid Speed of Sound & $a$ & 2490 & $\mathrm{~m} / \mathrm{s}$ & Tanks Diameter & $D_{t}$ & 52.9032 & $\mathrm{~m}$ \\
\hline \multirow{3}{*}{ Pipelines Lengths } & $L_{p_{1}}$ & 10326 & & & $\alpha_{1}$ & $1.93 \times 10^{-4}$ \\
& $L_{p_{2}}$ & 18560 & $\mathrm{~m}$ & Pipelines Inclinations & $\alpha_{2}$ & $3.77 \times 10^{-4}$ & $\mathrm{rad}$ \\
& $L_{p_{3}}$ & 14980 & & & & $\alpha_{3}$ & $5.47 \times 10^{-4}$ \\
\hline
\end{tabular}

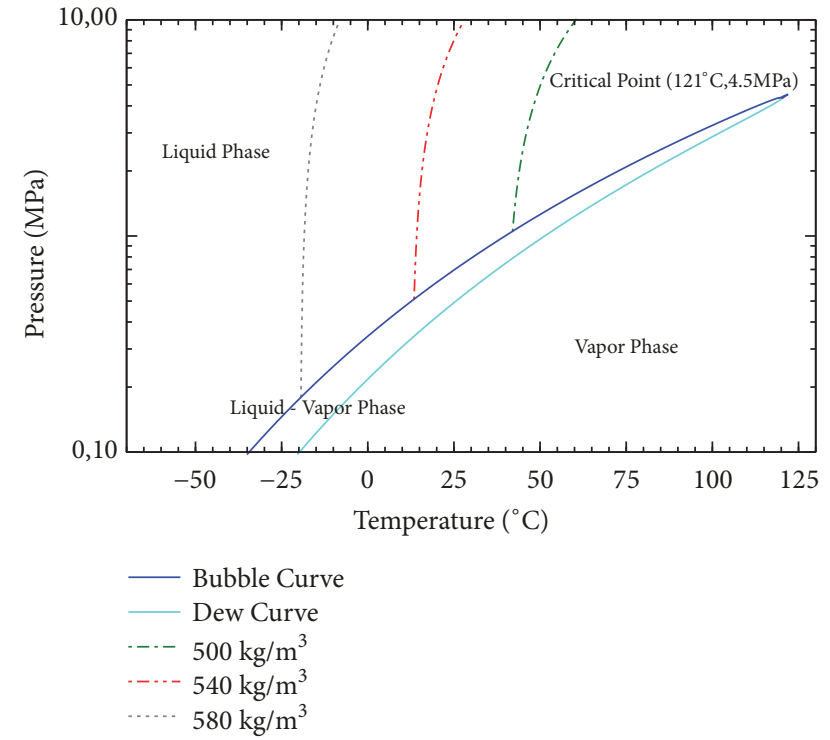

Figure 2: Pressure-Temperature Diagram of (40\% butane- $60 \%$ propane) LPG.

level of each storage tank $\left(C_{L}\right)$, by manipulating the pressure drop in pumps $\left(M_{P}\right)$ and control valves positions $\left(M_{v}\right)$, respectively, while respecting the physical limitations of the actuators. A set of discrete time Laguerre function is used as an orthonormal basis to describe the optimum future control trajectory (that is, the difference of the manipulated variable) within a prediction horizon. The use of the Laguerre function allows reducing the computational burden associated with MPC. Below the DMPC strategy is briefly presented.

3.1.1. Augmented State Space Model. As mentioned above, at the current study the MPC strategy used is based on state space models. The general extended state space model in (12) is employed. Recall that we embedded integrators in the state space model for obtaining integral action.

$$
\begin{aligned}
& \underbrace{\left[\begin{array}{c}
\Delta x_{p}(k+1) \\
e(k+1)
\end{array}\right]}_{x_{k+1}}=\underbrace{\left[\begin{array}{cc}
A_{p} & 0 \\
C_{p} A_{p} & 1
\end{array}\right]}_{A_{e}} \underbrace{\left[\begin{array}{c}
\Delta x_{p}(k) \\
e(k)
\end{array}\right]}_{x_{k}} \\
& +\underbrace{\left[\begin{array}{c}
B_{p} \\
C_{p} B_{p}
\end{array}\right]}_{B_{e}} \Delta u(k) \\
& e(k)=\underbrace{\left[\begin{array}{ll}
0 & 1
\end{array}\right]}_{C_{e}}\left[\begin{array}{c}
\Delta x_{p}(k) \\
y(k)
\end{array}\right]
\end{aligned}
$$

where subindex $p$ indicates process, $\Delta x_{p}(k+1)=x_{p}(k+$ $1)-x_{p}(k) \Delta u(k)=u(k)-u(k-1)$ and $e(k)=c^{\text {set }}(k)-c(k)$. Moreover, the poles of the new matrix are the poles of the process and one pole located at 1 . So, firstly it was confirmed the model was controllable and observable. Then, on the other hand, it was necessary to estimate the states, which are not measured; in this sense a Kalman filter was used [25], basically because, in the presence of noise and uncertainties, it produces state estimates that tend to be more precise. Kalman filter is commonly used for MPC vendor products to provide state estimation functionality [26]. Additionally, it is worth mentioning that the performance of the control strategies was evaluated by using the nonlinear pipeline model described by (1) and (2). By using the finite difference method, a spatialdiscrete version of the nonlinear model $[15,27]$ was obtained, which was used in closed loop simulations. This way, Figure 4 shows the block diagram of the implemented strategy, $G$ the process to be controlled, and $G_{C}$ the DMPC controller.

3.1.2. DMPC Formulation. The procedure established at [24] is centered on an orthonormal-base set of discrete functions to approximate the future differences of the manipulated 


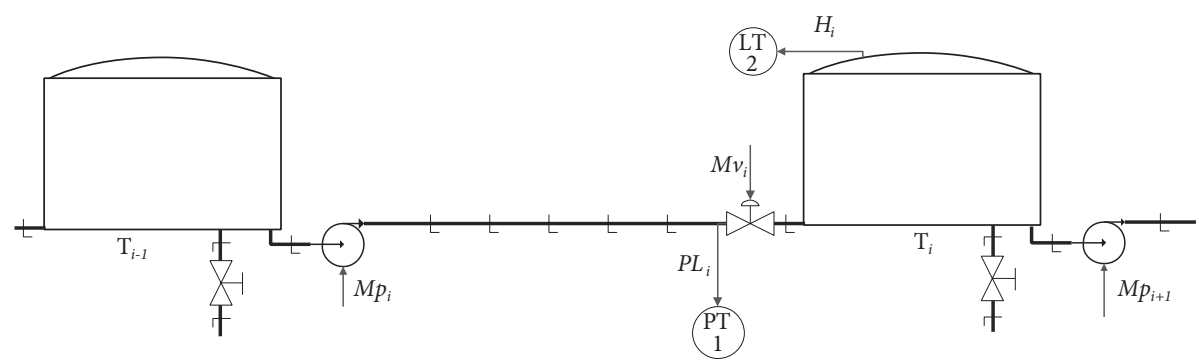

FIGURE 3: P\&ID for a pipeline section.

TABLE 2: Sensors and final control elements features.

\begin{tabular}{|c|c|c|c|}
\hline Instrument & Symbol & Value & Units \\
\hline \multirow{3}{*}{ Pumps } & $\Delta p_{\max }$ & 1 & $\mathrm{MPa}$ \\
\hline & $\tau_{p}$ & 30 & $\mathrm{~s}$ \\
\hline & $\bar{m}_{s}$ & 50 & $\%$ \\
\hline \multirow{2}{*}{ Pressure Transmitter } & span & 6 & $\mathrm{MPa}$ \\
\hline & $\tau_{P T}$ & 1 & $\mathrm{~s}$ \\
\hline \multirow{2}{*}{ Level Transmitter } & span & 20 & $\mathrm{~m}$ \\
\hline & $\tau_{L T}$ & $\approx 0$ & $\mathrm{~s}$ \\
\hline \multirow{5}{*}{ Valves } & $K_{v 1}$ & $6 \times 10^{-3}$ & $\mathrm{~m}^{3} /(\mathrm{s} . \%)$ \\
\hline & $K_{v 2}$ & $6 \times 10^{-3}$ & $\mathrm{~m}^{3} /(\mathrm{s} . \%)$ \\
\hline & $K_{v 3}$ & $2.8 \times 10^{-3}$ & $\mathrm{~m}^{3} /(\mathrm{s} . \%)$ \\
\hline & $\tau_{p}$ & 45 & $\mathrm{~s}$ \\
\hline & $\bar{m}$ & 50 & $\%$ \\
\hline
\end{tabular}

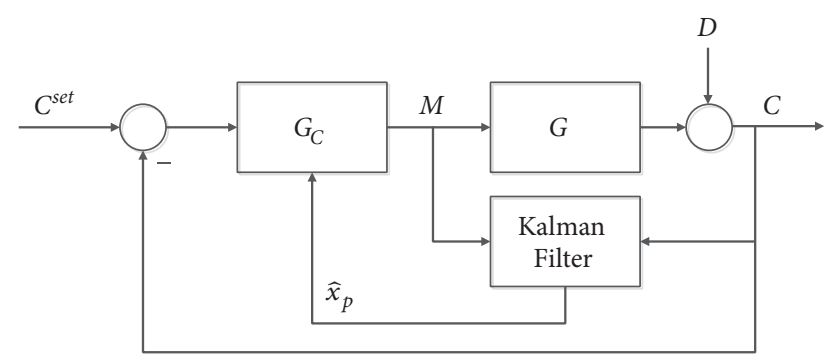

FIGURE 4: A general block diagram of the implemented strategy.

variable $\Delta m_{k_{i}+i}=\left[\begin{array}{llll}\Delta m_{k_{i}} & \Delta m_{k_{i}+1} & \cdots & \Delta m_{k_{i}+N_{c}-1}\end{array}\right]^{T}$, where $N_{C}$ indicates the control horizon. These functions are known as discrete Laguerre functions, and its z-transform is shown in (11).

$$
\begin{aligned}
& \Gamma_{k}(z)=\frac{z^{-1}-a}{1-a z^{-1}} \Gamma_{k-1}(z), \\
& \Gamma_{1}(z)=\frac{\sqrt{1-a^{2}}}{1-a z^{-1}}
\end{aligned}
$$

The pole of the function $a$ determines the velocity at which $l(k)$ converges to zero. Then, $\Delta m$ is approximated by $N$ Laguerre functions, such as in (12), where $k_{i}$ represents the current sampling period, $k$ are the future sampling periods, $N$ is the number of terms used at the expansion, and $c_{j}$ are the coefficients of the process which are the function of future sampling periods.

$$
\Delta m_{k_{i}+k}=\sum_{j=1}^{N} c_{j}\left(k_{i}\right) l_{j}(k)
$$

Laguerre functions can be expressed in the state space form in (13), which reduce computational burden. $A_{l}$ is a $N \times N$ matrix, which is a function of $a$.

$$
\begin{aligned}
& L(k+1)=A_{l} L(k), \\
& L(0)^{T} \\
& \quad=\sqrt{1-a^{2}}\left[\begin{array}{llllll}
1 & -a & a^{2} & -a^{3} & \cdots & (-1)^{N-1} a^{N-1}
\end{array}\right]^{T}
\end{aligned}
$$

Therefore (12) can also be represented as a function of the Laguerre vector defined at (13) such as

$$
\Delta m_{k_{i}+k}=L(k)^{T} \eta, \quad \eta=\left[\begin{array}{llll}
c_{1} & c_{2} & \cdots & c_{N}
\end{array}\right]^{T}
$$

Thus, the prediction of future states and outputs in an instant $m$ is defined by (15).

$$
\begin{aligned}
& x_{k_{i}+m \mid k_{i}}=A^{m} x_{k_{i}}+\sum_{i=0}^{m-1} A^{m-i-1} B L(i)^{T} \eta \\
& y_{k_{i}+m \mid k_{i}}=C x_{k_{i}+m \mid k_{i}}
\end{aligned}
$$




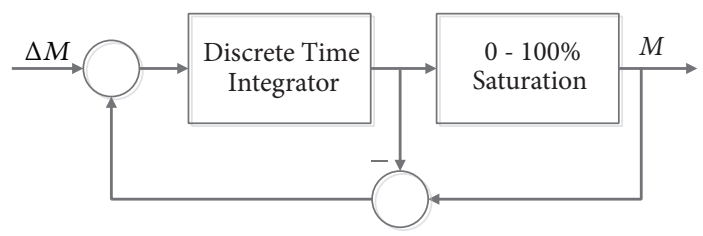

FIGURE 5: Constraint handling scheme using the antiwindup back-calculation method.

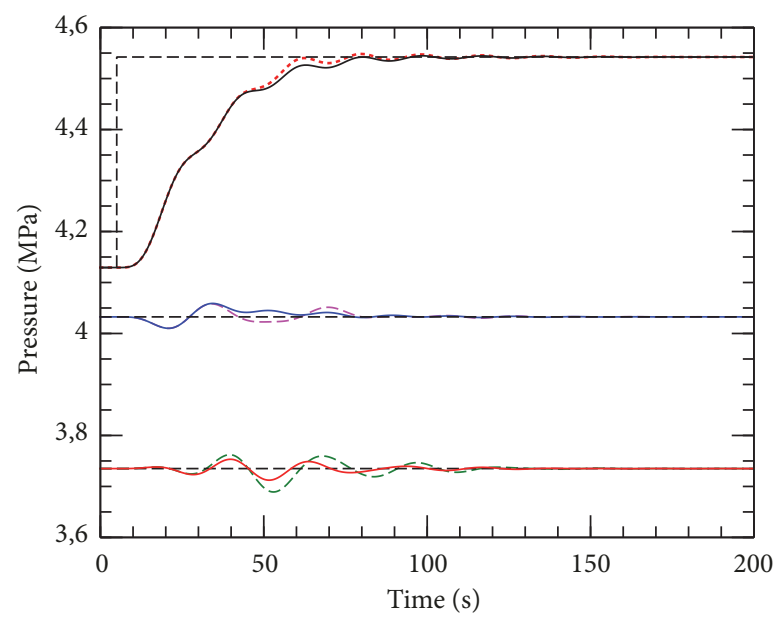

Station 1 (Const.) $\mathrm{IAE}=181.8$

Station 2 (Const.) IAE $=13.8$

Station 3 (Const.) $\mathrm{IAE}=13.59$

Station 1 (B-Calc.) $\mathrm{IAE}=177.3$

Station 2 (B-Calc.) $\mathrm{IAE}=14.74$

Station 3 (B-Calc.) $\mathrm{IAE}=22.96$
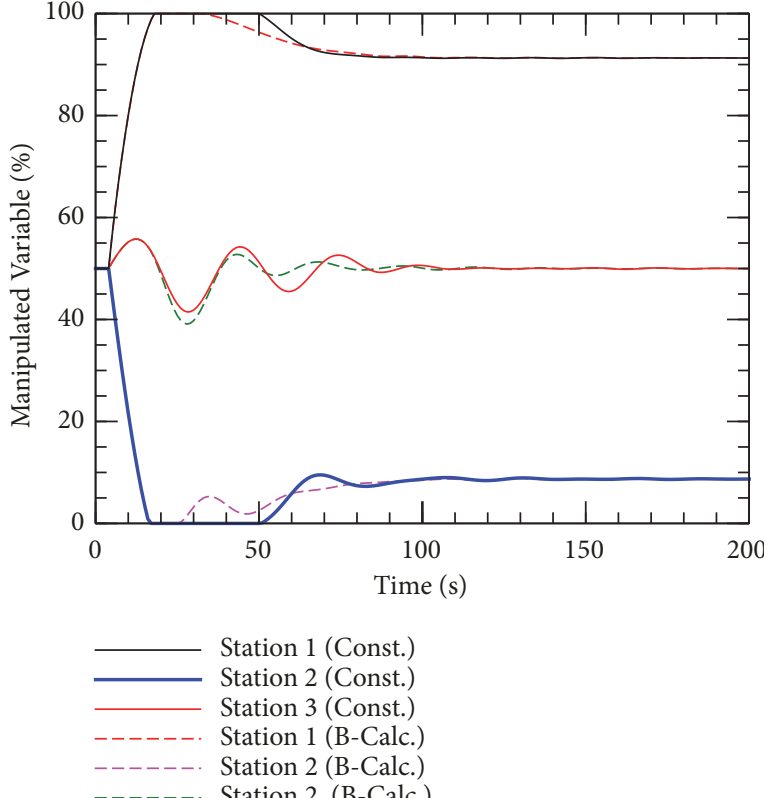

FIGURE 6: Performance comparison between the Constrain and the Back-Calculation methods.

Then, the cost function to be minimized can be written as (16), where $Q=C^{T} C$ and $R_{L}>0$. It is necessary to note $J$ is a function of $\eta$ instead of $\Delta M$, so minimization must be done in function of $\eta$.

$$
J=\sum_{m=1}^{N_{p}} x_{k_{i}+m \mid k_{i}}^{T} Q x_{k_{i}+m \mid k_{i}}+\eta^{T} R_{L} \eta
$$

Results of the minimization operation are shown in

$$
\begin{aligned}
\eta & =-\Omega^{-1} \Psi x\left(k_{i}\right) \\
\Omega & =\sum_{m=1}^{N_{p}} \phi(m) Q \phi(m)^{T}+R_{L} \\
\Psi & =\sum_{m=1}^{N_{p}} \phi(m) Q A^{m} \\
\phi(m)^{T} & =\sum_{i=0}^{m-1} A^{m-i-1} B L(i)^{T}
\end{aligned}
$$

Therefore, the control law is written at (18).

$$
\Delta m_{k}=k_{D M P C} \widehat{x}_{k}
$$

3.1.3. Constraint Handling. To take into account physical actuators limitations, a constraint is imposed on the control signal $\Delta m_{k}$, which is mathematically defined in

$$
m^{\min } \leq m_{k} \leq m^{\max }
$$

By using the Laguerre functions, it is possible to rewrite expressions in (19) as shown in

$$
m^{\min } \leq \sum_{i=0}^{k-1} L(i)^{T} \eta+m_{k-1} \leq m^{\max }
$$

Furthermore, it is important to note that constrained control strategies require real-time optimization using quadratic programming [24], where the minimization function would be (21). This restricted optimization must be done at the entire prediction horizon, which increases the computational burden.

$$
J=\eta^{T} \Omega \eta+2 \eta^{T} \Psi x_{k_{i}}
$$

Thus, at this research, a second option for constraining handling has been proposed which involves applying a backcalculation scheme, Figure 5. 


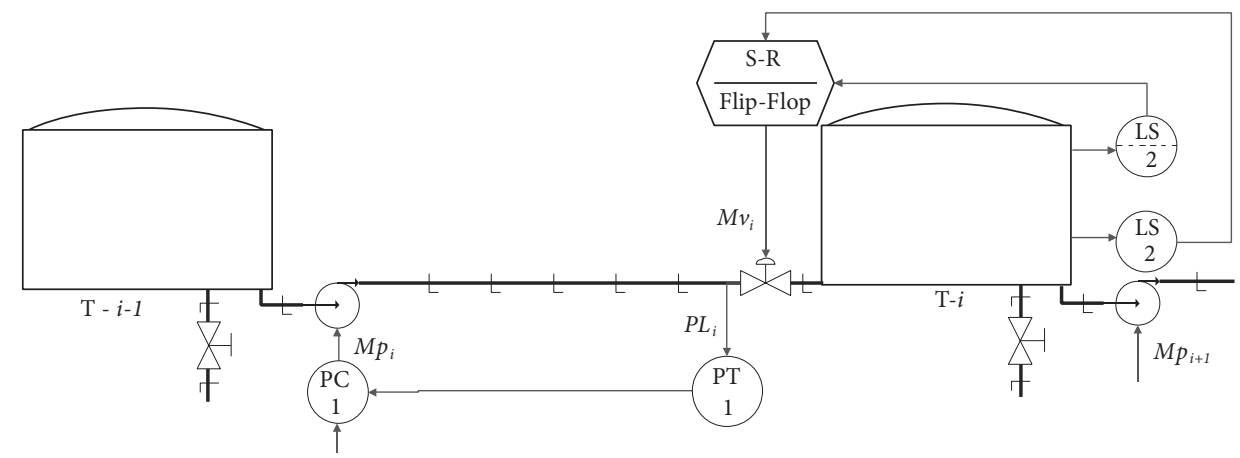

(a)

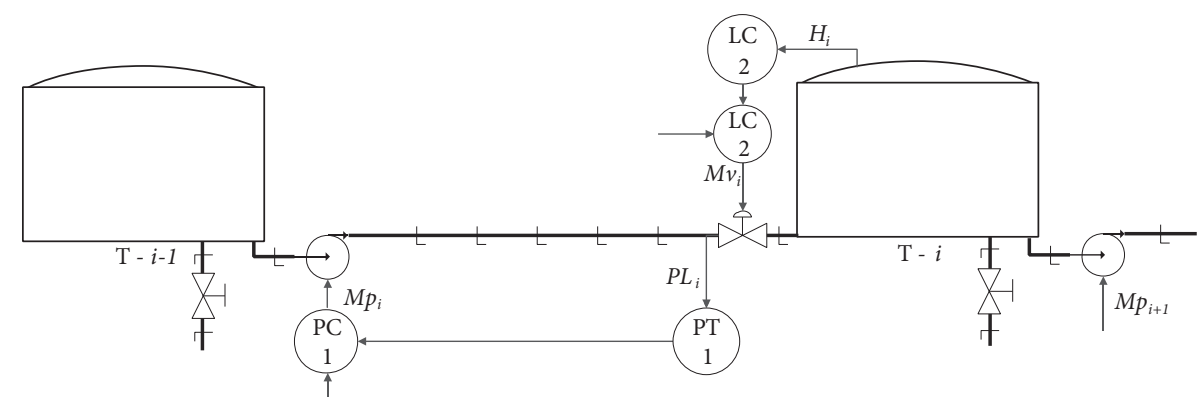

(b)

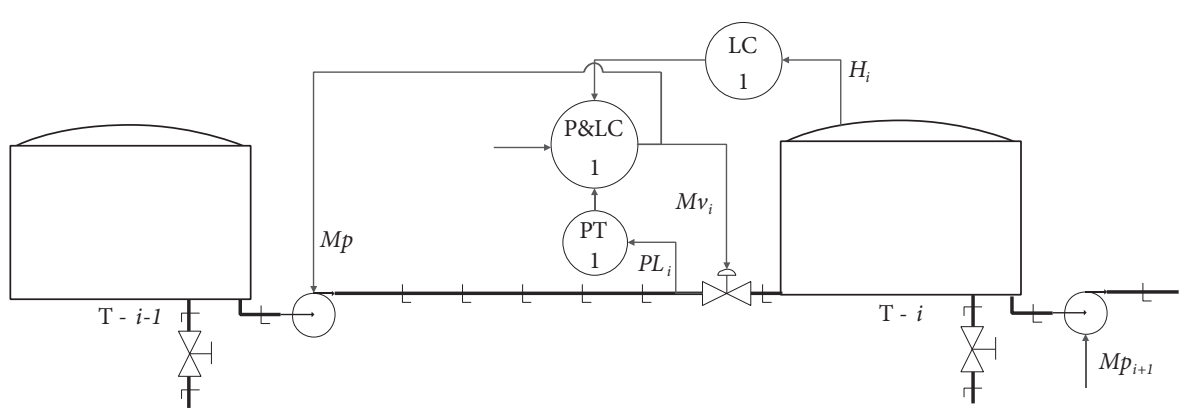

(c)

FIGURE 7: Pipeline Section P\&ID for (a) Configuration 1, (b) Configuration 2, and (c) Configuration 3.

Both methods were implemented, and simulations were run considering one change in the pressure set point of station 1. Results showed that both methods back-calculation and constraint handling based on quadratic optimization are very similar, as can be seen in Figure 6. As it refers to the IAE value, it is observed that the back-calculation method is slightly better than the constrain-handling method for the setpoint change in station 1 . However, even though the effect over this change over the other stations is low, the response of the optimization based constrain-handling method is somewhat better than the back-calculation method. So, provided both algorithms are equivalent, it was decided to use the method with the best performance from a computational point of view. In this case, the back-calculation method is much better than the optimization based constrainhandling method, having that it is approximately three times faster. Therefore, for this research, the back-calculation scheme was used. Some performance indices obtained with back-calculation scheme were overshoot of $0.1526 \%$, the rise time of 39.056s, and settling time of 67.805s.

\section{Results and Discussions}

Three different control configurations (see Figure 7) were implemented: (1) two decoupled control systems, a DMPC based control system for pressure, and an on-off control system for level, (2) two decoupled control systems for pressure and level based on DMPC strategy, and (3) one control system for pressure and level, in this configuration, the design model used is shown in (22). Table 3 shows the values of the controller's parameters used. The setpoint for level control was fixed in $7.89 \mathrm{~m}$ for the three tanks. 

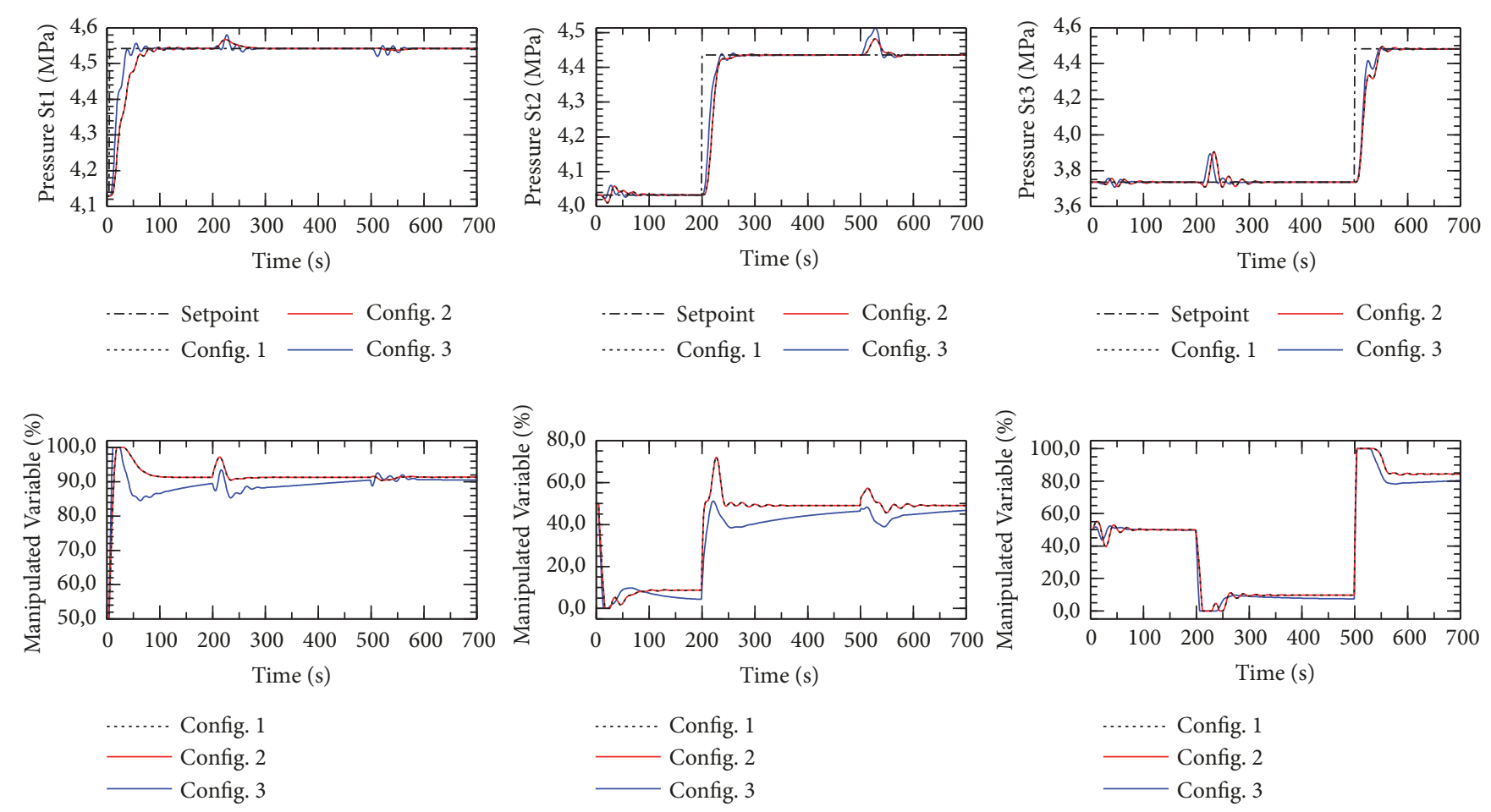

Figure 8: Pressure Response, under setpoint changes, for Configurations 1, 2, and 3.

Operating process conditions evaluated were pressure set points increments of $10 \%, 10 \%$, and $20 \%$ (at times 5, 200 and 500 seconds) for $P L_{1}, P L_{2}$, and $P L_{3}$, respectively, an increment of $50 \%$ (at 1000 seconds) in the output flow rate in tank 3 . The obtained performances are shown next.

$$
\begin{aligned}
& {\left[\begin{array}{c}
P L_{1} \\
P L_{2} \\
P L_{3} \\
H_{1} \\
H_{2} \\
H_{3}
\end{array}\right](s)} \\
& =\left[\begin{array}{lllllll}
G_{11} & G_{12} & G_{13} & G_{14} & G_{15} & G_{16} \\
G_{21} & G_{22} & G_{23} & G_{24} & G_{25} & G_{26} \\
G_{31} & G_{32} & G_{33} & G_{34} & G_{35} & G_{36} \\
G_{41} & G_{42} & G_{43} & G_{44} & G_{45} & G_{46} \\
G_{51} & G_{52} & G_{53} & G_{54} & G_{55} & G_{56} \\
G_{61} & G_{62} & G_{63} & G_{64} & G_{65} & G_{66}
\end{array}\right](s)\left[\begin{array}{l}
M_{p 1} \\
M_{p 2} \\
M_{p 3} \\
M_{v 1} \\
M_{v 2} \\
M_{v 3}
\end{array}\right](s)
\end{aligned}
$$

First response to be seen is the performance of the control strategy implemented under setpoint changes, so, in Figure 8 one step change to each controlled pressure is shown. Thus, as it is appreciated that a setpoint change on $P L_{1}$, let us call it $P L_{1}^{\text {set }}$, has a low impact on controlled pressured $P L_{2}$ and $P L_{3}$. Referring to a step change at $P L_{2}{ }^{\text {set }}$, it is shown that the effect on $P L_{3}$ is larger, and its disturbance to $P L_{1}$ is appreciable but smaller than change seen at $P L_{3}$. On the other hand, a change in $P L_{3}$ also has a low effect on $P L_{2}$ and almost an imperceptible effect on $P L_{1}$. Thus, even though the system is interacting, this interaction seems to be restricted to locally near the unit process. In other words, changes at station 1 have a significant effect on station 2 rather than 3 , changes at station 2 impact stations 1 and 3 in a similar way and changes at station 3 affect mainly over station 2 .

In Figure 8 it is seen that control strategies can maintain the process at its desired setpoint. The performance of configurations 1 and 2 is similar, as it was expected since the pressure controller used in both configurations is the same. Moreover, the interaction between stations is more prominent as they are closer. In what concerns configuration 3 , its performance is close to that showed by the other configurations; however, it looks its response velocity is higher. As it refers to tanks levels, pressure changes do not have an impact on them; basically level in tanks remained constant under pressure setpoints changes.

The strategies were also tested by producing a disturbance at the outlet flow rate at station 3. Thus, Figure 9 shows how, for configuration 1, this disturbance impacts the pressure of the other stations. As it was expected response at station 3 is more intense and, as it is seen, previous stations variations are still evident but lower. For configurations 1 and 2, variations in tanks levels due to disturbances cannot be seen, and constant levels are obtained.

Table 4 shows the IAE performance index obtained for each configuration, where it is definitively seen; configuration 3 gives the best results as it refers to IAE. 

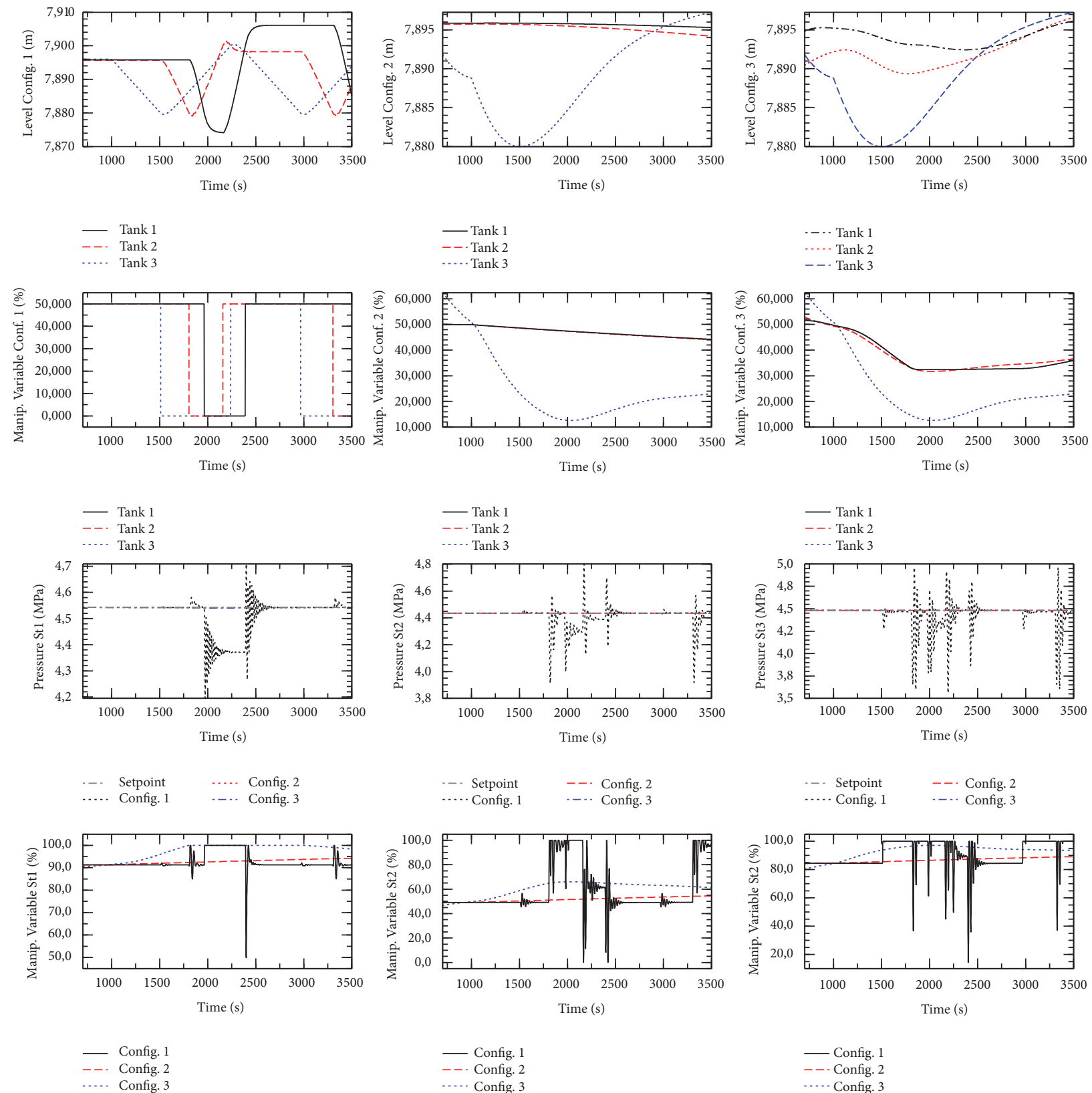

_Config. 1
--. Config. 2
… Config. 3

..... Config. 3

FIgURE 9: Level and Pressure Response, under disturbances, for Configurations 1, 2, and 3.

TABLE 3: Pressure and level control parameters.

\begin{tabular}{ccc}
\hline Configuration & Pressure Control Parameters & Level Control Parameters \\
\hline 1 & $a=\left[\begin{array}{lll}0.98 & 0.4 & 0.8\end{array}\right]$ & A S-R flip-flops were used, and lower and \\
$N=\left[\begin{array}{lll}2 & 2 & 9\end{array}\right]$ & higher levels were set to 7.88m and 7.9m, \\
respectively.
\end{tabular}


TABLE 4: IAE performance index obtained.

\begin{tabular}{lccr}
\hline Variable & Configuration 1 & Configuration 2 & Configuration 3 \\
\hline$P L_{1}$ & 1634 & 203.1 & 154.7 \\
\hline$P L_{2}$ & 1589 & 182.9 & 154.3 \\
\hline$P L_{3}$ & 3181 & 344.3 & 288.7 \\
\hline$H_{1}$ & $5.527 \times 10^{5}$ & 4.579 & 41.9 \\
\hline$H_{2}$ & $5.528 \times 10^{5}$ & 10.54 & 68.2 \\
\hline$H_{3}$ & $5.528 \times 10^{5}$ & 393.6 & 106.7 \\
\hline
\end{tabular}

\section{Conclusions}

Three control configurations were implemented for a liquid pipeline transportation system: (1) two decoupled control systems, an MPC based control system for pressure and an on-off control system for level, (2) two decoupled control systems for pressure and level based on MPC strategy, and (3) one MPC system for pressure and level. A linearized pipeline model was used as a design model; however, the performance of the control configurations was assessed with a nonlinear pipeline model. The addition of back-calculation as the antiwindup method allows reducing the control algorithms execution time significantly while maintaining the same performance as the achieved with the optimization based constraint handling method. In comparison with previous works [7], the strategies implemented show an enhanced performance. Lower IAE ( $0.1526 \%$ vs. $14.63 \%)$ and overshoot $\left(177.3\right.$ vs. $\left.8.4 \times 10^{5}\right)$ were obtained. Higher rise time and settling time was obtained with the proposed control strategies, but it is due to dimension, length $(10326 \mathrm{~m}$ vs. $7 \mathrm{~m})$ and diameter $(0.5 \mathrm{~m}$ vs. $0.0254 \mathrm{~m})$, of the pipeline considered. Among the three control configurations presented at the current study, the last one (Centralized Pressure and Level Control) showed the best performance among the different test carried out in the face of setpoints changes and disturbances. The IAE performance index confirmed this result.

\section{Nomenclature}

$\begin{array}{ll}a: & \text { Velocity of sound } \\ \lambda: & \text { Friction factor } \\ \alpha: & \text { Pipeline inclination } \\ L_{p}: & \text { Pipeline length } \\ P: & \text { Pressure } \\ V: & \text { Velocity } \\ \rho: & \text { Density } \\ \tau_{x x}: & \text { Normal stress respect to } x \\ k_{p}: & \text { Pump gain } \\ \tau_{p}: & \text { Pump time constant } \\ L_{p_{i}}: & \text { Section I pipeline length } \\ d: & \text { Pipeline diameter } \\ \alpha_{i}: & \text { Section } i \text { pipeline inclination } \\ H_{\text {max }}: & \text { Tank Height } \\ D_{t}: & \text { Tank Diameter } \\ P L_{i}: & \text { Section } i \text { outlet pressure } \\ M_{p_{i}}: & \text { Pump } i \text { pressure drop } \\ M_{v_{i}}: & \text { Valves position }\end{array}$

$G_{P L}: \quad$ Pipeline pressure model

$G_{L}: \quad$ Valves position model

$G_{D}: \quad$ Disturbances model

$Q_{k}$ : Covariance of the measurement error

$K_{k}: \quad$ Kalman gain

$\widehat{x}_{k}: \quad$ Estimated state

$P_{k}$ : $\quad$ Measurement error covariance estimated

$N_{c}$ : Horizon of control

$\Gamma_{i}: \quad$ Laguerre function

$l_{i}: \quad$ Inverse transform of $\Gamma_{i}$

$J_{S E}: \quad$ Quadratic cost function

$H(k)$ : Prediction using $l_{i}$

$\Delta m_{k}: \quad$ Control output

$K_{D M P C}$ : Control law

$A_{P}: \quad$ Pipe cross-sectional area.

\section{Data Availability}

Any additional information would be provided by the corresponding author via email.

\section{Conflicts of Interest}

The authors declare that they have no conflicts of interest.

\section{References}

[1] J. A. Jiménez Cabas, M. Sanjuán, and L. Torres, Liquid Transport Pipeline Monitoring Architecture Based on State Estimators for Leak Detection and Location, Universidad del Norte, 2018.

[2] R. Tubb, "P\&GJ's 2017 worldwide pipeline construction report," Pipeline \& Gas Journal, 2017.

[3] V. Thang Pham, D. Georges, G. Besançon et al., "Predictive control with guaranteed stability for hyperbolic systems of conservation laws," in Proceedings of the 49th IEEE Conference on Decision and Control (CDC), pp. 6932-6937, 2010.

[4] T. V. Pham, D. Georges, and G. Besancon, "Predictive control with guaranteed stability for water hammer equations," Institute of Electrical and Electronics Engineers Transactions on Automatic Control, vol. 59, no. 2, pp. 465-470, 2014.

[5] V. Yuzhanin, V. Popadko, T. Koturbash, V. Chernova, and R. Barashkin, "Predictive control and suppression of pressure surges in main oil pipelines with counter-running pressure waves," International Journal of Pressure Vessels and Piping, 2019.

[6] A. J. Osiadacz and M. Chaczykowski, "Dynamic control for gas pipeline systems," Archives of Mining Sciences, vol. 61, no. 1, pp. 69-82, 2016. 
[7] E. B. Priyanka, C. Maheswari, and S. Thangavel, "Online monitoring and control of flow rate in oil pipelines transportation system by using PLC-based Fuzzy-PID Controller," Flow Measurement and Instrumentation, vol. 62, pp. 144-151, 2018.

[8] M. Bauer and I. K. Craig, "Economic assessment of advanced process control-a survey and framework," Journal of Process Control, vol. 18, no. 1, pp. 2-18, 2008.

[9] X. Wang, B. Ding, X. Yang, and Z. Ye, "Design and application of offset-free model predictive control disturbance observation method," Journal of Control Science and Engineering, vol. 2016, Article ID 7279430, 8 pages, 2016.

[10] D. G. Vale da Fonseca, A. F. Dantas, C. E. Dórea, and A. L. Maitelli, "Explicit GPC control applied to an approximated linearized crane system," Journal of Control Science and Engineering, vol. 2019, Article ID 3612634, 13 pages, 2019.

[11] J. Duarte, J. Garcia, J. Jiménez, M. E. Sanjuan, A. Bula, and J. González, "Auto-ignition control in spark-ignition engines using internal model control structure," Journal of Energy Resource Technology, vol. 139, no. 2, p. 22201, 2017.

[12] M. Ławryńczuk, Computationally Efficient Model Predictive Control Algorithms, Springer, 2014.

[13] G. Lars and P. Jürgen, Nonlinear Model Predictive Control Theory and Algorithms, Springer, 2011.

[14] Y. G. Xi, D. W. Li, and S. Lin, "Model predictive control — status and challenges," Acta Automatica Sinica, vol. 39, no. 3, pp. 222236, 2013.

[15] J. Jiménez, L. Torres, I. Rubio, and M. Sanjuan, "Auxiliary signal design and liénard-type models for identifying pipeline," in Modeling and Monitoring of Pipelines and Networks: Advanced Tools for Automatic Monitoring and Supervision of Pipelines, C. Verde and L. Torres, Eds., pp. 99-124, Springer, 2017.

[16] J. Jimenez-Cabas, L. Torres, F. R. Lopez-Estrada, and M. Sanjuan, "Leak diagnosis in pipelines by only using flow measurements," in Proceedings of the IEEE Colombian Conference on Automatic Control (CCAC), 2017.

[17] L. Torres, G. Besançon, A. Navarro, O. Begovich, and D. Georges, "Examples of pipeline monitoring with nonlinear observers and real-data validation," in Proceedings of the 8th IEEE Int. Multi-Conf Signals Syst. Devices, pp. 1-6, 2011.

[18] J. Jiménez-Cabas, E. Romero-Fandiño, L. Torres, M. Sanjuan, and F. R. López-Estrada, "Localization of leaks in water distribution networks using flow readings," IFAC-PapersOnLine, vol. 51, no. 24, pp. 922-928, 2018.

[19] D. Matko, G. Geiger, and W. Gregoritza, "Pipeline simulation techniques," Mathematics and Computers in Simulation, vol. 52, no. 3-4, pp. 211-230, 2000.

[20] S. Blažič, D. Matko, and G. Geiger, "Simple model of a multibatch driven pipeline," Mathematics and Computers in Simulation, vol. 64, no. 6, pp. 617-630, 2004.

[21] J. F. Noguera and S. Leirens, "Modelling and simulation of a multi-commodity pipeline network," in Proceedings of the 2010 IEEE ANDESCON Conference, ANDESCON '10, pp. 1-6, 2010.

[22] J. J. Cabas and J. D. R. Ariza, "Modeling and simulation of a pipeline transportation process," Journal of Engineering and Applied Sciences, vol. 13, no. 9, 2018.

[23] L. Torres and C. Verde, "Modeling improvements for leak detection in pipelines of LPG," in Proceedings of the 2013 European Control Conference, ECC '13, pp. 938-942, 2013.

[24] L. Wang, "Discrete model predictive controller design using Laguerre functions," Journal of Process Control, vol. 14, no. 2, pp. 131-142, 2004.
[25] M. S. Grewal and A. P. Andrews, Kalman Filtering: Theory and Practice Using MATLAB, John Wiley \& Sons, 2011.

[26] S. J. Qin and T. A. Badgwell, "A survey of industrial model predictive control technology," Control Engineering Practice, vol. 11, no. 7, pp. 733-764, 2003.

[27] J. Jiménez, L. Torres, C. Verde, and M. Sanjuán, "Friction estimation of pipelines with extractions by using state observers," IFAC-PapersOnLine, vol. 50, no. 1, pp. 5361-5366, 2017. 


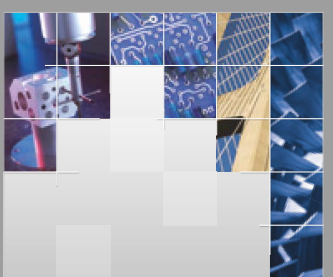

\section{Enfincering}
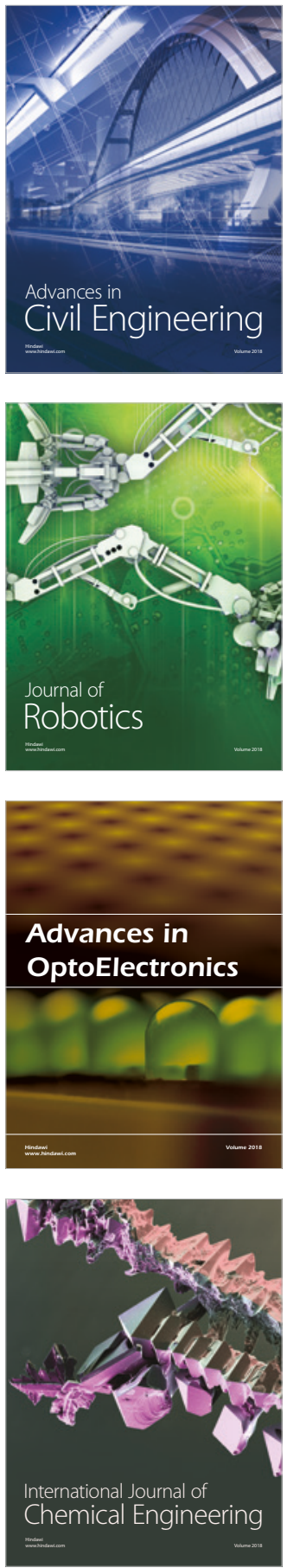

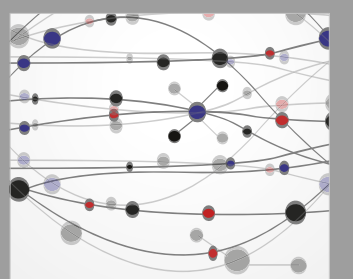

\section{Rotating \\ Machinery}

The Scientific World Journal

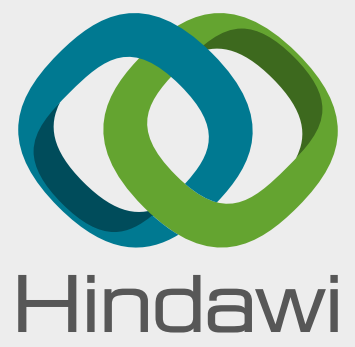

Submit your manuscripts at

www.hindawi.com
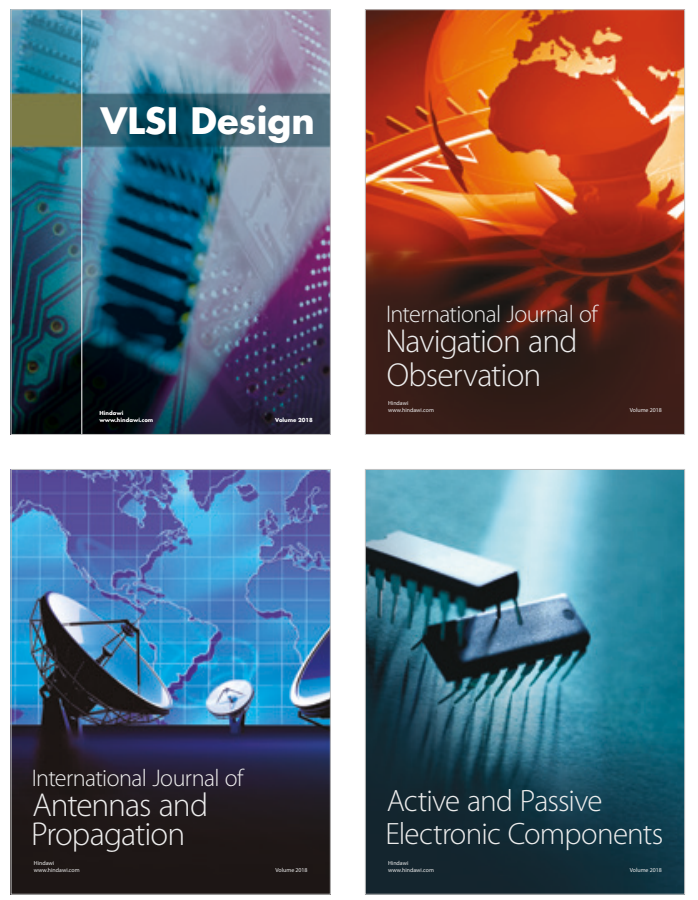
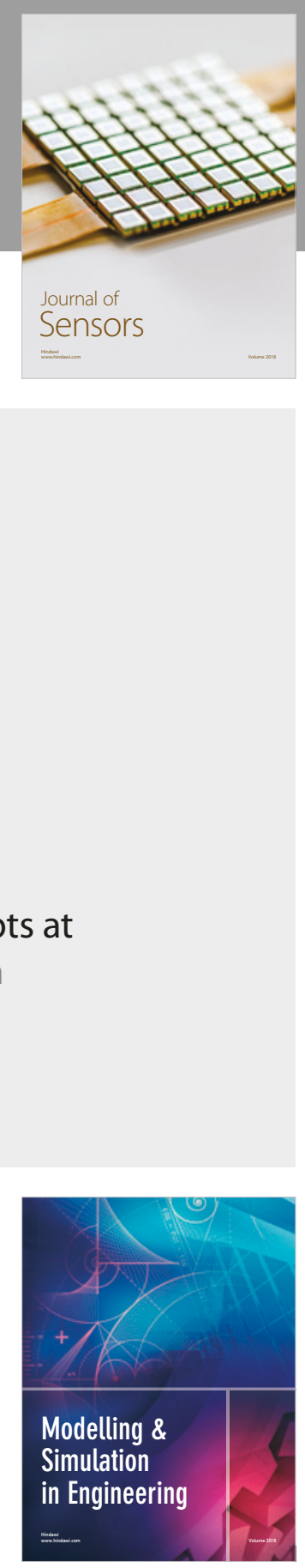

\section{Advances \\ Multimedia}
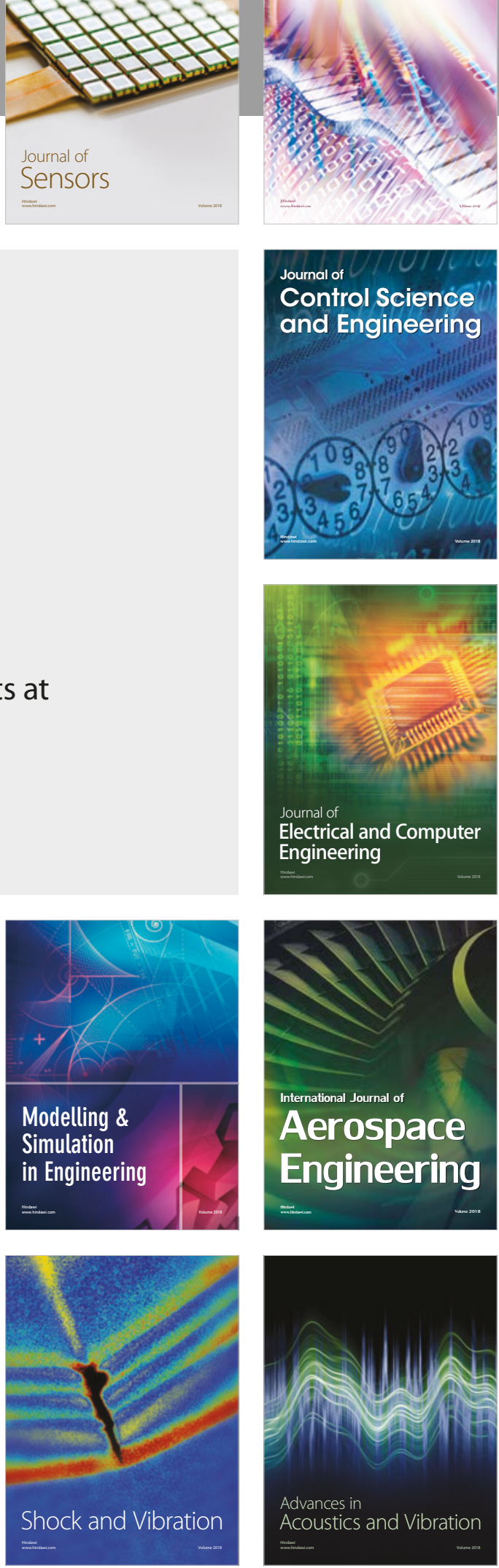